\title{
High Frequency Gravitational Radiation in Kerr-Schild Space-Times *
}

\author{
A. H. Taub \\ Mathematics Department, University of California, Berkeley, California, USA
}

\begin{abstract}
Vaidya has obtained general solutions of the Einstein equations $R_{a b}=\sigma \xi_{a} \xi_{b}$ by means of the Kerr-Schild metrics $g_{a b}=\eta_{a b}+H \xi_{a} \xi_{b}$. The vector field $\xi_{a}$ generates a shear free null geodetic congruence both in Minkowski space and in the Kerr-Schild space-time. If in addition it is hypersurface orthogonal, the Kerr-Schild metric may be interpreted as the "background metric" in a space-time perturbed by a high frequency gravitational wave. It is shown that Vaidya's solutions satisfying this additional condition are of only two types: (1) Kinnersley's accelerating point mass solution and (2) a similar solution where a space-like curve plays the role of the time-like curve describing the world line of the accelerating mass. The solution named by Vaidya as the radiating Kerr metric does not satisfy the hypersurface orthogonal condition.
\end{abstract}

\section{Introduction}

It is the purpose of this paper to apply the methods and results of Vaidya [1] and of MacCallum and Taub [2] to the discussion of high-frequency gravitational waves in Kerr-Schild space-times. The latter authors have described such waves and their gravitational effects by assuming that they produce a space-time whose metric tensor is given by

$$
g_{\mu \nu}\left(\varepsilon^{-1} X\right)=\stackrel{\circ}{g}_{\mu \nu}(X)+\varepsilon\left(\alpha_{\mu \nu}(X) e^{i \Psi(X) \varepsilon^{-1}}+\bar{\alpha}_{\mu \nu}(X) e^{-i \Psi(X) \varepsilon^{-1}}\right),
$$

where the bar over a quantity denotes the complex conjugate operation.

Thus they assume that the "background" metric, $\stackrel{\circ}{g}_{\mu \nu}(X)$, is a slowly varying function of coordinates and that the perturbation due to the gravitational wave, given by the coefficient of $\varepsilon$ in Eq. (1.1) is described by a slowly varying complex

\footnotetext{
* Supported in part by National Science Foundation Grant MPS 741029.
} 
amplitude $\alpha_{\mu \nu}$ and a rapidly varying phase. The vector

$$
l_{\mu}=\partial \Psi / \partial X^{\mu}
$$

determines the direction of rapid variation of various quantities.

If $g_{\mu \nu}$ given by Eq. (1.1) is to satisfy the Einstein vacuum field equations to second order in $\varepsilon$, and not arise from $\stackrel{\circ}{g}_{\mu \nu}$ by a coordinate transformation we must have

$$
\begin{aligned}
l_{\mu} l^{\mu} & =0, \\
a_{\mu v} v^{v} & =0,
\end{aligned}
$$

where

$$
\begin{gathered}
a_{\mu v}=\alpha_{\mu v}+a \stackrel{g}{\mu v}_{\mu v} / 2 \\
a=a_{\mu v} \stackrel{\circ}{g}^{\mu \nu}, \\
\left(\sqrt{-} \stackrel{g}{g} l^{\mu}\right),{ }_{\mu} \quad 0,
\end{gathered}
$$

where the comma denotes the partial derivative with respect to the variables $X^{\mu}$;

$$
N=a^{\sigma \tau} \bar{a}_{\sigma \tau}-\frac{1}{2} a \bar{a} \geqq 0
$$

and

$$
\stackrel{\circ}{G}^{\mu \nu}=\left(\varepsilon^{2} / 2\right) N l^{\mu} l^{\nu},
$$

where $\stackrel{\circ}{G}^{\mu v}$ is the Einstein tensor computed from $\stackrel{\circ}{\mu \nu}_{\mu \nu}(X)$, that is the "background" Einstein tensor.

Equations (1.2) and (1.3) imply that $l_{\mu}$ is a null, hyper-surface orthogonal, vector. Equation (1.6) is a conservation equation for the quantity $N$. Equation (1.8) describe the back reaction of the wave on the background metric $\stackrel{\circ}{g v}_{\mu v}$.

In this paper we shall assume that the background space time has a KerrSchild metric. That is we shall assume that

$$
\stackrel{\circ}{g}_{\mu \nu}=\eta_{\mu \nu}+H \xi_{\mu} \xi_{v},
$$

where $\eta_{\mu \nu}$ is the metric tensor of Minkowski space and $\xi_{\mu}$ is a null, geodesic and shear free vector field in both the Minkowski space and in the space-time with the metric given by Eq. (1.9).

The solutions of Eq. (1.8) subject to Eq. (1.9) that are needed for our discussion are a subset of those described by Vaidya and are obtained by imposing the additional condition that $\xi_{\mu}$ be hypersurface orthogonal. That is, that

$$
\varepsilon^{\mu \nu \sigma \tau} \xi \sigma, \tau \xi \nu=0
$$

where $\varepsilon^{\mu v \sigma \tau}$ is the Levi-Civita alternating tensor density.

It will be shown that this latter condition restricts the solutions to two types: (1) Kinnersley's accelerating point mass solution [3] and (2) a similar solution where a space-like curve plays the role of the time-like curve describing the world line of the accelerating mass. It is noteworthy that the solution that Vaidya labels as the radiating Kerr metric [4] does not satisfy Eq. (1.10) and hence cannot be interpreted by means of high-frequency gravitational wave perturbation. 
Since solutions of Types (1) and (2) determine Kerr-Schild space-times, there is an associated Minkowski space-time and a correspondence between shear free geodesics in these two space-times. The source of the gravitational field in the Kerr-Schild space-time may be said to be determined by a curve in the Minkowski space-time from which the congruence of null shear-free geodesics in the latter space-time emanates. On the image of this curve in the Kerr-Schild space-time, the metric becomes singular.

\section{Vaidya's Results}

These results, given in [1], may be conveniently described in terms of a tetrad of null vectors in Minkowski space. In an inertial coordinate system in this space we have

$$
\eta_{a b}=-\delta_{a b}+2 \delta_{a}^{4} \delta_{b}^{4} .
$$

We may write two real null vectors and a complex one as

$$
\begin{aligned}
& \xi=-\sin \alpha \cos \beta \partial / \partial x^{1}-\sin \alpha \sin \beta \partial / \partial x^{2}-\cos \alpha \partial / \partial x^{3}+\partial / \partial x^{4} \\
& \eta=\sin \alpha \cos \beta \partial / \partial x^{1}+\sin \alpha \sin \beta \partial / \partial x^{2}+\cos \alpha \partial / \partial x^{3}+\partial / \partial x^{4} \\
& M=-(\cos \alpha \cos \beta+i \sin \beta) \partial / \partial x^{1}-(\cos \alpha \sin \beta-i \cos \beta) \partial / \partial x^{2}+\sin \alpha \partial / \partial x^{3} .
\end{aligned}
$$

Then

$$
\xi^{a} \eta_{a}=-M^{a} \bar{M}_{a}=2
$$

and all other scalar products formed from this tetrad of vectors vanish. Further

$$
\eta_{a b}=\frac{1}{2}\left(\xi_{a} \eta_{b}+\xi_{b} \eta_{a}\right)-\frac{1}{2}\left(M_{a} \bar{M}_{b}+\bar{M}_{a} M_{b}\right) .
$$

We also define the vector

$$
A_{a}=\frac{1}{2}\left(\alpha_{, a}+i \beta_{, a} \sin \alpha\right),
$$

where the comma denotes the partial derivative with respect to the $x^{a}$ s.

It follows that the condition that $\xi^{a}$ be a geodesic, shear-free vector field is equivalent to the requirement that

$$
\xi^{a} A_{a}=\bar{M}^{a} A_{a}=0
$$

as may be verified from the fact that

$$
\xi_{b, a}=A_{a} M_{b}+\bar{A}_{a} \bar{M}_{b}=-\eta_{b, a}
$$

and

$$
M_{b, a}=-\bar{A}_{a} \xi_{b}+\bar{A}_{a} \eta_{b}+\cot \alpha\left(A_{a}-\bar{A}_{a}\right) M_{b} .
$$

We define

$$
\begin{aligned}
& \theta=\xi^{a},{ }_{a} \\
& \text { and } \\
& \Omega^{2}=\left(\xi_{a, b}-\xi_{b, a}\right) \xi^{a},{ }_{c} \eta^{c b} .
\end{aligned}
$$


Then we have

$$
z=\theta-i \Omega=2 A^{a} M_{a} .
$$

Vaidya's geometric parameters $V$ and $W$ and two other useful parameters may be defined as follows:

$$
\begin{aligned}
& u=x^{a} \xi_{a} \\
& v=x^{a} \eta_{a} \\
& \gamma=V+i W=x^{a} M_{a} .
\end{aligned}
$$

Hence

$$
\begin{aligned}
& u_{,_{b}}=\xi_{b}+\gamma A_{b}+\bar{\gamma} \bar{A}_{b} \\
& v_{,_{b}}=\eta_{b}-\gamma A_{b}-\bar{\gamma} \bar{A}_{b}
\end{aligned}
$$

and

$$
\gamma_{, b}=M_{b}+\gamma \cot \alpha A_{b}+(v-u-\gamma \cot \alpha) \bar{A}_{b} .
$$

It is a consequence of the above results that $\alpha, \beta, u$, and $\gamma$ are solutions of the equations

$$
\xi^{a} f, a=0 .
$$

We take $\alpha, \beta$, and $u$ as independent solutions and regard

$$
\gamma=\gamma(u, \alpha, \beta) \text {. }
$$

In this case

$$
\gamma_{, b}=\gamma_{u} u_{, b}+\gamma_{\alpha}\left(A_{b}+\bar{A}_{b}\right)-i \operatorname{cosec} \alpha \gamma_{\beta}\left(A_{b}-\bar{A}_{b}\right),
$$

where

$$
\gamma_{u}=\partial \gamma / \partial u \quad \gamma_{\alpha}=\partial \gamma / \partial \alpha \quad \gamma_{\beta}=\partial \gamma / \partial \beta
$$

On equating the two expressions for $\gamma_{, b}$ we find that we must have when $z \neq 0$

$$
\begin{aligned}
& \gamma \gamma_{u}+\gamma_{\alpha}-\gamma \cot \alpha-i \gamma_{\beta} \operatorname{cosec} \alpha=0 . \\
& \bar{\gamma} \gamma_{u}+\gamma_{\alpha}+\gamma \cot \alpha+i \gamma_{\beta} \operatorname{cosec} \alpha+u-v=-4 z / z \bar{z} \\
& A_{c} \eta^{c}=\bar{\gamma}_{u} A_{c} M^{c} .
\end{aligned}
$$

It then follows that

$$
A_{a}=(z / 4)\left(\bar{\gamma}_{u} \xi_{a}-\bar{M}_{a}\right) \text {. }
$$

We may then write

$$
\begin{aligned}
\xi_{b} & =u_{, b}-\gamma A_{b}-\bar{\gamma} \bar{A}_{b} \\
\eta_{b} & =v_{, b}+\gamma A_{b}+\bar{\gamma} \bar{A}_{b} \\
M_{b} & =\gamma_{u} \xi_{b}-4 z \bar{A}_{b} / z \bar{z}
\end{aligned}
$$

and thus readily express the metric of space-time in terms of the variables $u, v, \alpha$, and $\beta$, when $\gamma$ and $z$ are known as functions of $u, \alpha$, and $\beta$. 
The space-time with the metric tensor

$$
g_{a b}=\eta_{a b}+H \xi_{a} \xi_{b}
$$

where $\xi_{a}$ is defined as above has been shown in [1] to have

$$
R_{a b}=\sigma \xi_{a} \xi_{b}
$$

provided $\gamma$ and $z$ are related by Eq. (2.2),

$$
H=-M(z+\bar{z}) / 2=-M \theta
$$

and $M$ is a real function of $u, \alpha, \beta$ above such that

$$
\gamma M_{u}+M_{\alpha}-i M_{\beta} \operatorname{cosec} \alpha+3 \gamma_{u} M=0 .
$$

In this case

$$
\sigma=\frac{1}{2} M_{u} z \bar{z} .
$$

The two real equations determining $M$ may be written as

$$
\begin{aligned}
& V M_{u}+3 M V_{u}+M_{\alpha}=0 \\
& W M_{u}+3 M W_{u}-M_{\beta} \operatorname{cosec} \alpha=0 .
\end{aligned}
$$

When neither of these equations is satisfied identically as a result of restrictions imposed on the quantities $M, V, W$, the integrability conditions of these equations leads to the result

$$
M=f(\alpha, \beta) p^{-3},
$$

where

$$
p=V W_{u}+W_{\alpha}=i(z-\bar{z}) / z \bar{z} .
$$

In addition one must have

$$
\left[(1 / p)\left(W_{u} V_{\beta}-W V_{\beta u}+W^{2} V_{u u} \sin \alpha-p_{\beta}\right)\right]_{u}=0 .
$$

Given $\gamma(u, v, \beta)$ satisfying the first of Eq. (2.2) we may invert Eq. (2.1) and express $x^{a}$ as functions of $u, v, \alpha, \beta$. The curves of parameter $v$ are the curves of the null, geodesic, shear-free congruence determined by the vector field $\xi^{a}$. The values of $\theta$ and $\Omega$ for this null congruence are determined by the second of Eq. (2.2). Equation (2.3) enable one to readily express the line element in Minkowski space-time in terms of the coordinates $u, v, \alpha, \beta$. The function $M$ satisfying Eq. (2.6) for given $\gamma$ determines $M$ and hence the Kerr-Schild metric such that Eq. (2.4) are satisfied with $\sigma$ given by Eq. (2.5).

\section{The Condition $\Omega=0$}

In order to carry out the program outlined in the introduction we must restrict the vector field $\xi_{a}$ so that Eq. (1.13) and their equivalents, Eq. (1.14) are satisfied. These equations are in turn equivalent to the statement that

$$
\Omega=i(z-z) / 2=0 \text {. }
$$


Thus we must determine a complex valued function $\gamma$ such that

$$
\begin{array}{r}
\gamma \gamma_{u}+\gamma_{\alpha}-\gamma \cot \alpha-i \gamma_{\beta} \operatorname{cosec} \alpha=0 \\
(\gamma+\bar{\gamma})\left(\gamma_{u}-\bar{\gamma}_{u}\right)+2\left(\gamma_{\alpha}-\bar{\gamma}_{\alpha}\right)=0,
\end{array}
$$

and then determine the real function $M(u, \alpha, \beta)$ satisfying Eq. (2.4).

If we write

$$
\gamma=V+i W,
$$

Eq. (3.3) becomes

$$
V W_{u}+W_{\alpha}=0 .
$$

It is convenient to write

$$
\theta=(z+\bar{z}) / 2
$$

and hence

$$
1 / \theta=-\frac{1}{2}\left[V V_{u}+V_{\alpha}+\frac{1}{2}(u-v)\right] \text {. }
$$

In terms of the variables $V$ and $W$ Eq. (3.2) may be written as

$$
\begin{gathered}
V V_{u}-W W_{u}+V_{\alpha}-V \cot \alpha+W_{\beta} \operatorname{cosec} \alpha=0, \\
W V_{u}+V W_{u}+W_{\alpha}-W \cot \alpha-V_{\beta} \operatorname{cosec} \alpha=0 .
\end{gathered}
$$

We shall limit our discussion of the solutions to Eqs. (3.4), (3.6), and (3.7) to the situation when $V_{\beta}=0$. In that case it follows from the first and third of these equations that

$$
W\left(V_{u}-\cot \alpha\right)=0
$$

and hence we have either

Case $\mathrm{A}: W=0$

or

Case B : $V_{u}=\cot \alpha$ or both.

Case A has been treated by Vaidya [5] and has been shown to consist of his radiating star metric, Robinson and Trautman metric and Kinnersley's accelerating point mass metric. In Case A Eq. (3.6) becomes

$$
V V_{u}+V_{\alpha}-V \cot \alpha=0
$$

and admits the solution $V=0$. Equation (2.4) then state that $M$ is an arbitrary function of $u$. Equation (3.5) becomes

$$
\theta=(v-u) / 4
$$

and the line element becomes that denoted by Vaidya as the radiating star metric [4]. 
Another special solution is given by

$$
V=-u \tan \alpha \text {. }
$$

Equation (2.4) becomes

$$
M_{u}+x M_{x}=-3 M
$$

where $x=\cos \alpha$. Hence $M$ is a homogeneous function of degree -3 in the variables $u$ and $\cos \alpha$. Equation (3.4) becomes

$$
1 / \theta=-\frac{1}{2}\left[V \cot \alpha+\frac{1}{2}(u-v)\right]=(u+v) / 4 .
$$

The general solution of Eq. (3.8) is given by

$$
V=\sin \alpha h(g),
$$

where $h$ is an arbitrary non-constant function,

$$
g=u+V \cot \alpha=u+h(g) \cos \alpha
$$

and hence

$$
V g_{u}+g_{\alpha}=0
$$

since

$$
\begin{aligned}
& g_{u}=\left(1-h^{\prime}(g) \cos \alpha\right)^{-1} \\
& g_{\alpha}=-\left(1-h^{\prime}(g) \cos \alpha\right)^{-1} h(g) \sin \alpha=-V g_{u} .
\end{aligned}
$$

It follows that

$$
1 / \theta=-\frac{1}{2}\left[g-\frac{1}{2}(u+v)\right]
$$

and

$$
M=m(g)\left(1-h^{\prime}(g) \cos \alpha\right)^{-3},
$$

where the prime denotes the derivative of $h(g)$ with respect to its argument and $m(g)$ is an arbitrary differentiable function of $g$.

In the next section we shall see the relation between the metric obtained when $W=0$ and $V$ is given by Eq. (3.12) to that given by Kinnersley.

In Case $\mathrm{B}$, when $V_{\beta}=0$, it is no restriction to choose constants of integration such that the general solution to Eqs. (3.4) and (3.6) is given by

$$
\begin{aligned}
u & =K(\omega) \sin \alpha \sin (\omega-\beta) \\
V & =K(\omega) \cos \alpha \sin (\omega-\beta)=u \cot \alpha \\
W & =K(\omega) \cos (\omega-\beta) .
\end{aligned}
$$

Then

$$
\theta=2 / t=4 /(u+v)
$$

and

$$
M=m(\omega) \varrho^{-3},
$$


where

$$
\varrho=\sin \alpha\left[K^{\prime} \sin (\omega-\beta)+K \cos (\omega-\beta)\right]
$$

and $K^{\prime}$ denotes the derivative of $K(\omega)$ with respect to its argument.

In Section 5 it will be shown that the metrics determined by the above expressions for $V$ and $W$ are similar to those given by Kinnersley. They differ in that a time-like curve which plays a key role in the latter metrics is replaced by a space-like one.

\section{The Radiation Energy Density, Case A}

The results of the preceding section together with Eq. (2.3) enable us to determine the Kerr-Schild metric in a coordinate system in which the variables $(\alpha, \beta, v, u)$ are regarded as the coordinates of an event in space-time. This follows from the fact that

$$
\eta_{a b} d x^{a} d x^{b}=\left(\xi_{a} d x^{a}\right)\left(\eta_{b} d x^{b}\right)-\left(M_{a} d x^{a}\right)\left(\bar{M}_{b} d x^{b}\right)
$$

and Eq. (2.3) allow one to readily calculate the right hand side of this equation. The resulting Kerr-Schild metric

$$
d s^{2}=\eta_{a b} d x^{a} d x^{b}-M \theta\left(\xi_{a} d x^{a}\right)^{2}
$$

satisfies the field equations

$$
R_{a b}=\sigma \xi_{a} \xi_{b}=-\frac{1}{2} M_{u}\left(\theta^{2}+\Omega^{2}\right) \xi_{a} \xi_{b} .
$$

Since we have imposed the condition that $\Omega=0$ we may write

$$
\xi_{a}=\varrho l_{a},
$$

where $l_{a}$ is the gradient of a scalar. If we identify the inertial coordinates $x^{a}$ used in Sections 2 and 3 with the variables $X^{a}$ used in Section 1, and the $g_{a b}$ of the former sections with $\stackrel{\circ}{g}_{a b}$ of Section 1 we see that

$$
\varrho^{2} \theta^{2} M_{u}=N .
$$

The conserved quantity $\frac{1}{2} \varepsilon^{2} N$ is interpreted as the energy density of the highfrequency radiation as measured by an observer with four velocity $u^{a}$ such that $u^{a} l_{a}=1$.

In this section we shall determine $\varrho$ for Case A when Eq. (3.12) hold. We have from Eq. (2.3)

$$
\xi_{b}=u_{, b}-V \alpha_{, b}=u_{, b}-h(g) \sin \alpha \alpha_{, b} .
$$

It follows from Eqs. (3.15) and (3.16) that

$$
g_{, b}=g_{u} u_{, b}+g_{\alpha} \alpha_{, b}=\left(1-h^{\prime}(g) \cos \alpha\right)^{-1}\left(u_{, b}-V \alpha_{, b}\right)
$$

that is,

$$
\xi_{b}=\left(1-h^{\prime}(g)\right) \cos \alpha g_{, b} .
$$


If we define

$$
r=\frac{1}{2}(u+v)-g
$$

we use Eq. (2.3) to determine $\xi_{a} d x^{a}, \eta_{a} d x^{a}$ and $M_{a} d x^{a}$. We also have

$$
H\left(\xi_{a} d x^{a}\right)^{2}=-2 m(g)(d g)^{2} /\left(1-h^{\prime}(g) \cos \alpha\right) r
$$

since

$$
\theta=2 / r, \quad M=m(g) /\left(1-h^{\prime}(g) \cos \alpha\right)^{-3} .
$$

It is evident that the variables $\alpha, \beta, r, g$ may be used as coordinates instead of $\alpha, \beta, u, v$.

It is of interest to write the inverses of Eq. (2.1) and express the inertial coordinates in Minkowski space in terms of the variables $\alpha, \beta, r, g$. We find that these equations may be written as

$$
x^{a}=r \xi^{a}+Y^{a}(g)
$$

where

$$
Y^{a}(g)=-h \delta_{3}^{a}+g \delta_{4}^{a}
$$

and is the world line of a particle moving on the $x^{3}$ axis. Thus the Kerr-Schild metric we are discussing is the one given by Kinnersley [3] for an accelerating point mass as has been pointed out by Vaidya in [4].

Kinnersley has used as coordinates a set of variables $(\alpha, \beta, R, \tau)$ instead of $(\alpha, \beta, r, g)$. The variables $R$ and $\tau$ have a direct geometrical interpretation in Minkowski space and are related to $r$ and $g$ as follows: $\tau$ is the proper-time along the world line given by $Y^{\mu}(g)$ and hence is related to $g$ by the equation

$$
d \tau / d g=\left(1-h^{\prime 2}\right)^{1 / 2} .
$$

The quantity $\tau$ may be considered as a function of $x^{a}$ by virtue of the fact that

$$
\eta_{a b}\left(x^{a}-Y^{a}(\tau)\right)\left(x^{b}-Y^{b}(\tau)\right)=0 .
$$

Then

$$
\tau_{, a}=\left(x_{a}-Y_{a}(\tau)\right) / R,
$$

where

$$
R=\left(d Y^{b} / d \tau\right)\left(x_{b}-Y_{b}(\tau)\right) .
$$

The equations of the null geodesics emanating from the world line $Y^{\mu}(\tau)$ are then given by

$$
x^{\mu}=R \eta^{\mu \nu} \tau, v+Y^{\mu}(\tau)
$$

and $R$ is the affine parameter along a particular null geodesic.

If we set

$$
l_{a}=\tau_{, a}
$$


and

$$
\varrho=\left(1-h^{\prime} \cos \alpha\right)\left(1-h^{\prime 2}\right)^{-1 / 2} .
$$

Equation (4.2) becomes

$$
\xi_{b}=\varrho l_{b} .
$$

Equations (4.4) and (4.6) become identical if we let

$$
R=r\left(1-h^{\prime} \cos \alpha\right)\left(1-h^{\prime 2}\right)^{-1 / 2} .
$$

It follows from the values of the various quantities involved that

$$
M_{u}=m^{\prime}\left(1-h^{\prime} \cos \alpha\right)^{-4}+3 m h^{\prime \prime} \cos \alpha\left(1-h^{\prime} \cos \alpha\right)^{-5} .
$$

If we now define

$$
\mu=m(g)\left(1-h^{\prime 2}\right)^{-3 / 2}
$$

and consider $g$ as a function of $\tau$, we may write Eq. (4.1) as giving

$$
N=-\left(4 / R^{2}\right)\left(\dot{\mu}-3 \mu \ddot{Y}^{a} \tau_{, a}\right)
$$

for the energy density of the radiation, where $Y^{a}$ is given by (4.5); the dot refers to differentiation with respect to $\tau$. In terms of these variables the line element takes the form given by Kinnersley, namely

$$
g_{\mu \nu}=\eta_{\mu \nu}-(2 \mu / R) \tau_{, \mu} \tau_{, v} .
$$

In case $h^{\prime}$ is a constant, that is, the particle is not accelerating, we may introduce a new set of variables $\left(\alpha^{*}, \beta, R, \tau\right)$ instead of $(\alpha, \beta, R, \tau)$ in terms of which the line element (4.7) becomes Vaidya's radiating star metric. We require $\left|h^{\prime}\right|<1$ and then the angle $\alpha$ is defined in terms of $\alpha$ by the relation

$$
\sin \alpha^{*}=\sqrt{1-h^{\prime 2}} \sin \alpha /\left(1-h^{\prime} \cos \alpha\right)
$$

thus

$$
\cos \alpha^{*}=\left(\cos \alpha-h^{\prime}\right) /\left(1-h^{\prime} \cos \alpha\right)
$$

and

$$
R \sin \alpha^{*}=r \sin \alpha .
$$

Equations (4.4) become

$$
x^{* a}=R \xi^{a}\left(\alpha^{*}\right)+Y^{* a},
$$

where the starred quantities are related to the unstarred ones by the Lorentz transformation which carries the world line given by Eq. (4.5) into the time axis and $\xi^{a}\left(\alpha^{*}\right)$ is obtained from $\xi^{a}$ by substituting $\alpha^{*}$ for $\alpha$.

It may be verified from Eq. (4.3) that we may write the line element for Case A when $h^{\prime}$ is not necessarily constant as

$$
\begin{aligned}
d s^{2}= & 2\left(1+\dot{h}^{2}\right)^{1 / 2} d\left(R\left(1+\dot{h}^{2}\right)^{-1 / 2}\right) d \tau+d \tau^{2}(1-2 \mu / R) \\
& -r^{2}\left(d \alpha^{2}+\sin ^{2} \alpha d \beta^{2}\right),
\end{aligned}
$$


where $h$ is considered as a function of $\tau$, the dot refers to differentiation with respect to $\tau, \mu$ is an arbitrary differentiable function of $\tau$ and

$$
R=r\left(\left(1+\dot{h}^{2}\right)^{1 / 2}-\dot{h} \cos \alpha\right) .
$$

Note that when $\dot{h}=0$ and $2 \mu<R$, the curves of parameter $\tau$ are timelike in the space-time with the line-element given by Eq. (4.9).

\section{The Radiation Energy Density, Case B}

The discussion of this case can be carried out along the lines used in the discussion of the preceding section. We begin with the evaluation of the inverses of Eq. (2.1) when Eq. (3.19) hold. It follows from Eq. (3.19) that

$$
x^{a}=t \xi^{a}+Y^{a}(\omega),
$$

where

$$
Y^{a}(\omega)=K(\omega) \sin \omega \delta_{1}^{a}-K(\omega) \cos \omega \delta_{2}^{a}
$$

and are the coordinates of a point on a space-like curve in the $x^{1}, x^{2}$ plane. Equation (5.1) are evidently similar to Eq. (4.4); they do, however, involve a space-like curve instead of one which is time-like when $h^{\prime}<1$ in the latter equations.

It follows from Eqs. (2.3) and (3.19) through (3.22) that

$$
\begin{aligned}
\xi_{a} d x^{a}= & \varrho d \omega \\
\eta_{a} d x^{a}= & 2 d t-\varrho d \omega \\
M_{a} d x^{a}= & {\left[\left(K^{\prime} \cos \alpha \sin (\omega-\beta)+K \cos \alpha \cos (\omega-\beta)\right) d \omega-t d \alpha\right] } \\
& +i\left[\left(K^{\prime} \cos (\omega-\beta)-K \sin (\omega-\beta)\right) d \omega+t \sin \alpha d \beta\right],
\end{aligned}
$$

where $\varrho$ is given by Eq. (3.22). If $\tau$ denotes the arc length along the curve given by Eq. (5.2) we have

$$
d \tau / d \omega=\left(K^{\prime 2}+K^{2}\right)^{1 / 2} .
$$

When we set

$$
T=t \varrho\left(K^{\prime 2}+K^{2}\right)^{-1 / 2}
$$

Eq. (5.1) may be written as

$$
x^{a}=T \eta^{a b} \tau_{, b}+Y^{a}(\tau)
$$

the direct analogues of Eq. (4.6). Equations (3.2) and (3.21) then give

$$
H\left(\xi_{a} d x^{a}\right)^{2}=M \theta\left(\xi_{a} d x^{a}\right)^{2}=(2 \mu / T) d \tau^{2},
$$

where

$$
\mu=m(\omega)\left(K^{\prime 2}+K^{2}\right)^{-3 / 2} .
$$

The metric tensor is then

$$
g_{a b}=\eta_{a b}+(2 \mu / T) \tau_{, a} \tau_{, b},
$$


where $\eta_{a b} d x^{a} d x^{b}$ may be calculated from Eq. (5.3). The energy density $N$ is again determined from Eq. (4.1) and a calculation leads to the equation analogous to (4.7) namely

$$
N=-\left(4 / T^{2}\right)\left(\dot{\mu}-3 \mu \ddot{Y}^{a} \tau_{, a}\right),
$$

where the dot refers to the differentiation with respect to the arc length along the curve given by Eq. (5.2), that is with respect to the variable $\tau$.

It follows from Eq. (5.3) and the above discussion that we may write the line element in this case as

$$
\begin{aligned}
d s^{2}= & 2\left(1-\dot{K}^{2}\right)^{1 / 2} K^{-1} d \tau d\left(T K\left(1-\dot{K}^{2}\right)^{-1 / 2}\right) \\
& -(1-2 \mu / T) d \tau^{2}-t^{2}\left[d \alpha^{2}+\sin ^{2} \alpha d \beta^{2}\right],
\end{aligned}
$$

where

$$
T=t \sin \alpha\left[\dot{K} \sin (\omega-\beta)-\left(1-\dot{K}^{2}\right)^{1 / 2} \cos (\omega-\beta)\right]
$$

and $K$ is a function of $\tau, \dot{K}$ is its derivative with respect to its argument $\mu$ and $\omega$ are functions of $\tau$. The latter is determined by Eq. (5.4) which may be written as

$$
d \tau / d \omega=K\left(1-\dot{K}^{2}\right)^{-1 / 2} .
$$

We observe that when $\dot{K}=0$, the curves $T=$ constant, $\alpha=$ constant, $\beta=$ constant arc space-like curves provided $T>2 \mu$ in contrast to the situation that pertains in Case A when $\dot{h}=0$.

\section{References}

1. Vaidya, P. C.: Proc. Camb. Phil. Soc. 75, 383-390 (1914)

2. MacCallum, M. A.H., Taub, A.H.: Commun. math. Phys. 30, 153-169 (1973)

3. Kinnersley, W.: Phys. Rev. 186, 1335-1336 (1969)

4. Vaidya, P.C., Patel,L. K.: Phys. Rev. D. 7, 3540 (1973)

5. Vaidya, P.C.: Tensor, N.S. 24, 315-321 (1972)

Communicated by J. Ehlers 